\title{
CURRENT FLUCTUATIONS IN FERROMAGNETIC TUNNEL JUNCTIONS
}

\author{
B.R. Bulka ${ }^{a}$, J. MartineK ${ }^{a}$, G. Michaeek ${ }^{a}$ AND J. Barnaś ${ }^{b}$ \\ ${ }^{a}$ Institute of Molecular Physics, Polish Academy of Sciences \\ Smoluchowskiego 17, 60-179 Poznań, Poland \\ ${ }^{b}$ Department of Physics, A. Mickiewicz University \\ Umultowska 85, 61-614 Poznań, Poland
}

\begin{abstract}
Current shot noise in a double junction consisting of a small metallic grain connected to two ferromagnetic electrodes is analysed in the limit of sequential tunneling. We show that, apart from charge fluctuations, there are strong spin fluctuations. We also show that two distinct relaxation processes can be distinguished in the frequency dependent current noise: one in a low frequency range and corresponding to spin fluctuations and another one in a high frequency range corresponding to charge fluctuations.
\end{abstract}

PACS numbers: 75.70.Pa, 73.50.Td, 73.23. Hk, 73.40.Gk

Recent interest in spin-polarized electron tunneling in magnetic junctions is stimulated by expected applications in microelectronic devices. A special kind of tunnel junctions are ferromagnetic single-electron transistors (FM SETs), which consist of a small metallic grain coupled via two tunnel junctions to ferromagnetic electrodes. A very important parameter, in view of potential applications of FM SETs, is the noise-to-signal ratio. The noise can also provide additional information on the electronic structure, transport properties and also on electron-electron interactions. In the case of non-magnetic single-electron transistors (NM SETs) this problem was studied extensively both theoretically [1] and experimentally [2]. However, the situation in ferromagnetic junctions significantly differs from that in NM SETs and to our best knowledge there are neither experimental nor theoretical works published up to now on the shot noise in FM SETs.

We analyse the shot noise of tunneling current in ferromagnetic double nanojunctions in the limit of incoherent sequential tunneling. In order to include the spin degrees of freedom, we extended the generation-recombination approach for multielectron channels by generalizing the method developed for spinless electrons in SETs [1] (details will be published elsewhere [3]). Figures 1a and b show the voltage $V$ dependence of the current $I$ and the zero-frequency current shot noise $S_{I I}(0)$ for the antiparallel and parallel orientations of the magnetisation in the electrodes. We additionally assumed there that the grain is small, with the energy level spacing $\Delta E \ll k_{\mathrm{B}} T$. The discreteness of the electronic structure of the grain 
is seen in Fig. 1 as the small steps on the curves. The reduction of $S_{I I}(0)$ below the value $S_{\text {Poisson }}=2 e I$ indicates that correlations between tunneling events are significant. The maximum reduction occurs at the steps of the $I-V$ curves, where new charge channels open for electron tunneling. This situation is common in all SETs. The current noise in the parallel configuration is larger than in the antiparallel one. This results from different levels of spin noise in both cases. In the antiparallel configuration there is some spin accumulation on the grain, which shrinks the space of available states for spin fluctuations and lowers the current noise as well.

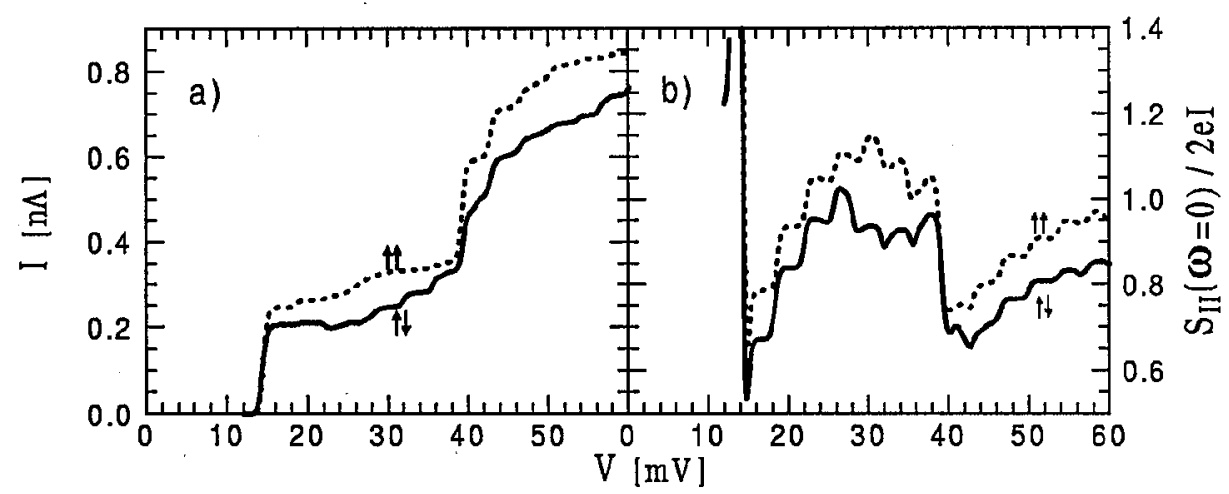

Fig. 1. Voltage dependences of the current (a) and the zero-frequency power noise (b) in the antiparallel and the parallel configurations (solid and dashed curves, respectively). The parameters assumed here are: the tunnel resistances $-R_{2 \uparrow}=240 \mathrm{M} \Omega, R_{2 \downarrow}=$ $60 \mathrm{M} \Omega, R_{1 \uparrow}=2 \mathrm{M} \Omega$, and $R_{1 \downarrow}=8 \mathrm{M} \Omega$ for the antiparallel configuration $\left(R_{2 \uparrow}=\right.$ $240 \mathrm{M} \Omega, R_{2 \downarrow}=60 \mathrm{M} \Omega, R_{1 \uparrow}=8 \mathrm{M} \Omega$, and $R_{1 \downarrow}=2 \mathrm{M} \Omega$ for the parallel configuration), the capacitances $-C_{2}=6.6 \mathrm{aF}, C_{1}=1.32 \mathrm{aF}$, the level spacing $-\Delta E=3 \mathrm{meV}$, and the temperature $-T=2.3 \mathrm{~K}$. The index $1(2)$ at the resistances and capacitances corresponds to the right (left) junction and the electrons flow from right to left.

In FM SETs the tunneling events with opposite spins occur with different frequencies and they are correlated. There are charge and spin fluctuations induced by flowing currents, which have a significant influence on the current noise and its frequency dependence. The power spectrum is written as $S_{I I}(\omega)=S_{I I}^{\mathrm{Sch}}+S_{I I}^{\mathrm{c}}(\omega)$, where $S_{I I}^{S c h}$ is the Schottky value (independent of $\omega$ ) and $S_{I I}^{\mathrm{c}}(\omega)$ is the frequency dependent part, with $S_{I I}^{\mathrm{c}} \rightarrow 0$ for $\omega \rightarrow \infty$. Figure 2 presents the frequency dependence of $S_{I I}^{\mathrm{C}}$ for different voltages. One can clearly see (e.g. for the curve corresponding to $V=26 \mathrm{mV}$ ) two relaxation processes contributing to the current noise: respectively in low and high frequency ranges. One can expect that charge and spin fluctuations contribute to the current noise and their relaxation times are in the range of the maximal slope of the curves in Fig. 2. Therefore, we determined the charge-charge and the spin-spin correlation functions, $S_{N N}(\omega)$ and $S_{M M}(\omega)$, respectively. The relaxation times corresponding to the charge and spin fluctuations are in a high and in a low frequency range and they are close to those ones determined from the slope of $S_{I I}^{c}(\omega)$ (at the voltages close to the $I-V$ steps the sit- 


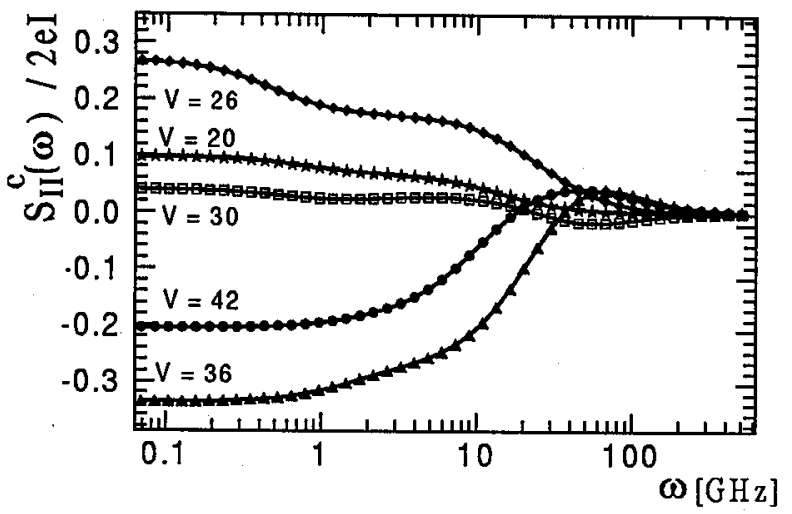

Fig. 2. Frequency dependence of the current shot noise $S_{I I}^{c}(\omega)$ in the system defined in Fig. 1 for the antiparallel configuration.
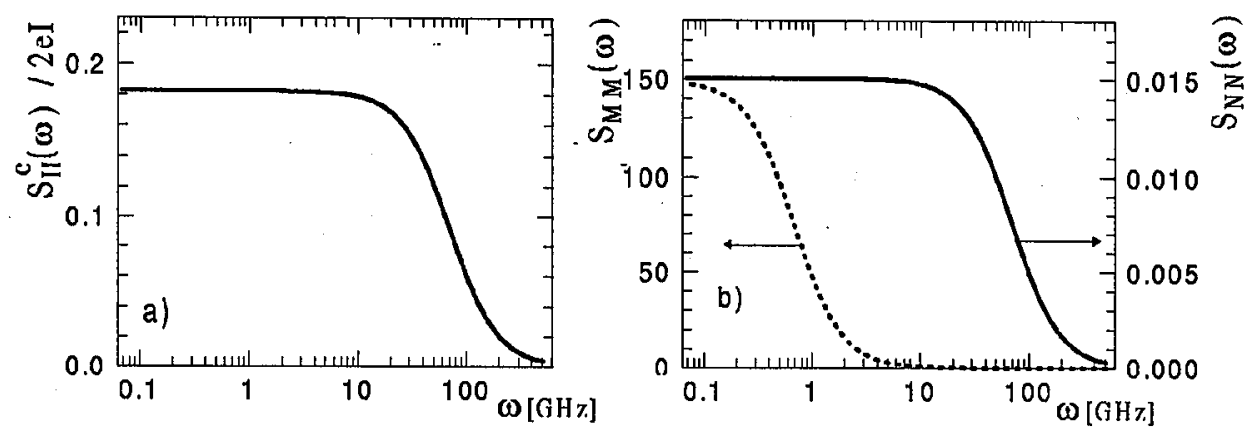

Fig. 3. Frequency dependence of the current shot noise (a), and of the spin (dashed curve) and charge (solid curve) (b) noise in a non-magnetic SET, calculated for $V=$ $26 \mathrm{mV}$. The other parameters assumed here are: $R_{1 \uparrow}=R_{1 \downarrow}=2 \mathrm{M} \Omega, R_{2 \uparrow}=R_{2 \downarrow}=$ $60 \mathrm{M} \Omega, C_{2}=6.6 \mathrm{aF}, C_{1}=1.32 \mathrm{aF}, \Delta E=3 \mathrm{meV}$, and $T=2.3 \mathrm{~K}$.

uation is more complex, since different charge channels have different relaxation times). The amplitude of spin fluctuations, defined as $\operatorname{var}(M)=\left\langle M^{2}\right\rangle-\langle M\rangle^{2}$, is one order of magnitude larger than the amplitude for the charge fluctuations $\operatorname{var}(N)=\left\langle N^{2}\right\rangle-\langle N\rangle^{2}$, where $N$ and $M$ are the excess numbers of electrons and spins on the grain. This is an effect of relatively small energy for opening a new magnetic channel $\Delta E=3 \mathrm{meV}$ and large charging energy $E_{c}=10.1 \mathrm{meV}$. Figure 2 shows additionally that the tunneling events can be anticorrelated in some cases, which results in $S_{I I}^{\mathrm{c}}(\omega)<0$. This can be seen for the curves corresponding to $V=36 \mathrm{mV}$ and $42 \mathrm{mV}$, where the amplitude of $S_{I I}^{\mathcal{C}}$ is large and negative in the range of small frequencies. In these cases the spin fluctuations are responsible for reduction of the current noise.

Spin fluctuations occur also in NM SETs, but they were neglected in the previous works [1]. Figure 3 explains the situation in the non-magnetic system. The current shot noise (Fig. 3a) does not show any low frequency component, 
despite of the presence of large spin fluctuations (see the dashed curve in Fig. 3b). In the case considered their amplitude is four orders in magnitude larger than that for charge fluctuations. In NM SETs the tunneling events with the frequency corresponding to spin fluctuations are anticorrelated for electrons with the same spin and correlated for electrons with the opposite spin. This leads to compensation of the spin component in the total current noise for each junction in NM SETs.

In summary, we have performed theoretical analysis of the frequency dependent current shot noise in ferromagnetic tunnel junctions in the limit of sequential tunneling. We have shown that apart from charge fluctuations, there are also strong spin fluctuations which influence the current noise. The spin noise is significant at low frequencies, while the charge noise at high frequencies. Accordingly, two distinct relaxation times should be seen in frequency measurements of the current noise in magnetic tunnel junctions.

The paper is supported by the Committee for Scientific Research under grant No. 2 PO3B 07514.

\section{References}

[1] A.N. Korotkov, Phys. Rev. B 49, 10381 (1994); S. Hershfield, J.D. Davies, P. Hyldgaard, C.J. Stanton, J.W. Wilkins, Phys. Rev. B 47, 1967 (1993); U. Hanke, Y.M. Galperin, K.A. Chao, N. Zou, Phys. Rev. B 48, 17209 (1993); U. Hanke, Y.M. Galperin, K.A. Chao, N. Zou, ibid. 50, 1595 (1994); A. Imamoglu, Y. Yamamoto, Phys. Rev. Lett. 70, 3327 (1993).

[2] H. Birk, M.J.M. de Jong, C. Schonenberger, Phys. Rev. Lett. 75, 1610 (1995).

[3] B.R. Bułka, J. Martinek, G. Michałek, J. Barnaś, preprint available on http://xxx.lanl.gov, cond-mat/9904341, to be published. 